\title{
Numerical Simulation of Slinky-coil Ground Heat Exchangers Installed in Railway Tunnels
}

\section{Hikari Fujii}

\author{
Satoko Taniguchi
}

\author{
Keisuke Ogai
}

\begin{abstract}
The use of horizontal ground heat exchangers (HGHEs) greatly reduces the initial cost of GSHP systems in comparison with vertical GHEs. Though the HGHEs are commonly used in North American countries, the installation number of the HGHEs is still few in European and Asian countries due to the limited land availability. The use of tunnels is one of the measures to promote the HGHEs because the tunnel walls are suitable for laying the HGHEs. In this research, analysis of operation data and numerical modeling were carried out in a GSHP system using Slinky-coil HGHEs installed in the basement of a railway tunnel in Tokyo. Through the interpretation of operation data, the stable energy efficiency of the system was confirmed, though the amount of heat disposal and extraction were not well-balanced in the GSHP system. A numerical simulation model of the HGHE was then developed based on the physical properties of ground and the design of the HGHEs. The simulation model was well-validated with the operation data and showed that 1) the GSHP system could maintain the initial heating and cooling capacity for 10 years and 2) the deeper installation of the HGHE is favorable to obtain higher COP.
\end{abstract}

\section{INTRODUCTION}

Today, vertical and horizontal ground heat exchangers (GHEs) are commonly used in ground source heat pump (GSHP) systems. Though horizontal ground heat exchangers (HGHEs) require large land space for installation, the installation cost of HGHEs are lower than that of vertical GHEs since the HGHEs can be installed using common excavation machines. For this reason, HGHEs are widely installed in USA and Canada, where more land space tends to be available than in European and Asian countries. Considering the low installation cost, the use of HGHE can improve the competitiveness of GSHP systems, in case the required surface land space is minimized.

Several researches have been carried out by European researchers in the last decade to use the underground space for HGHEs. Energy Geo-Structure (EGS) is a prefabricated panel containing horizontal heat exchangers inside which can be set on the walls of subway or railway tunnels (e.g., Bourne et al., 2016). Fordl et al. (2010) investigated the optimum design and installation method of EGS in the Brenner Base Tunnel in the Austrian Alps. Nicholson et al. (2013) studied in temperature performance and the stress distribution change with the operation of EGS in a subway tunnel in the U.K. Bourne-Webb et al. (2016) investigated the effect of the temperature, wind velocity in the tunnel and the thermal conductivity of the ground on the heat exchange performance in GSHP system with EGS using a FEM model. Barla et al. (2016) evaluated the energy saving and the environmental impact of EGS in a subway tunnel in Milan using a FEM model.

In Japan, GSHP systems using the tunnel walls for the installation of HGHEs are not introduced yet. In 2013, on the other hand, a GHSP system using HGHE installed in the basement of a railway tunnel was completed in Tokyo. Since the system uses only the basement of the tunnel, the construction procedure is less complicated and the impact on the tunnel wall strength is smaller than the sidewall installation. The system started the heating and cooling operations in July 2013 and the operation data have been recorded since then. In this research, the heat exchange Hikari Fujii (fujii@mine.akita-u.ac.jp) is a professor of energy resource engineering at Akita University, Japan. Satoko Taniguchi and Keisuke Ogai are GSHP engineers at Mitsubishi Materials Techo Corporation, Japan. 
performance, the load behavior and the ground temperature performance are evaluated based on the operation data. Then a numerical model of the HGHE and the surrounding ground is developed using a groundwater and thermal transport simulator, FEFLOW ver.7.0. The model is validated through a history matching with operation data and sensitivity studies are performed using the model to optimize the design and operation strategy of the HGHEs.

\section{INFORMATION ON THE GSHP SYSTEM}

The GSHP system using HGHEs was constructed in 2013 at Setagaya-Daita station in Tokyo. The station was relocated from land surface to underground to mitigate the traffic jams at railway crossings. Figure 1 shows the cross sectional view of the station. The passenger's platform is located in the B2 level (B2F), while express trains pass the B3 level (B3F). Five water-air heat pumps (cooling/heating capacity: $5.0 \mathrm{~kW} / 5.2 \mathrm{~kW}$, respectively) are installed in B2F to supply the warm and cool air to the passenger's waiting room on the platform.

The HGHEs are installed in the basement concrete of the B3F. The depth of HGHE from the surface of the basement and land surface are $1.9 \mathrm{~m}$ and $26.6 \mathrm{~m}$, respectively. The HGHEs are Slinky coils of $0.8 \mathrm{~m}$ diameter and 0.6 $\mathrm{m}$ pitch using high-density polyethylene tubes of $24 \mathrm{~mm} / 34 \mathrm{~mm}$ ID/OD. The thermal conductivity of the concrete is enhanced using SiO2-rich aggregates. The geology of the ground is loam and the thermal conductivity of ground and concrete were measured as $1.39 \mathrm{~W} / \mathrm{m} / \mathrm{K}$ and $2.34 \mathrm{~W} / \mathrm{m} / \mathrm{K}$, respectively.

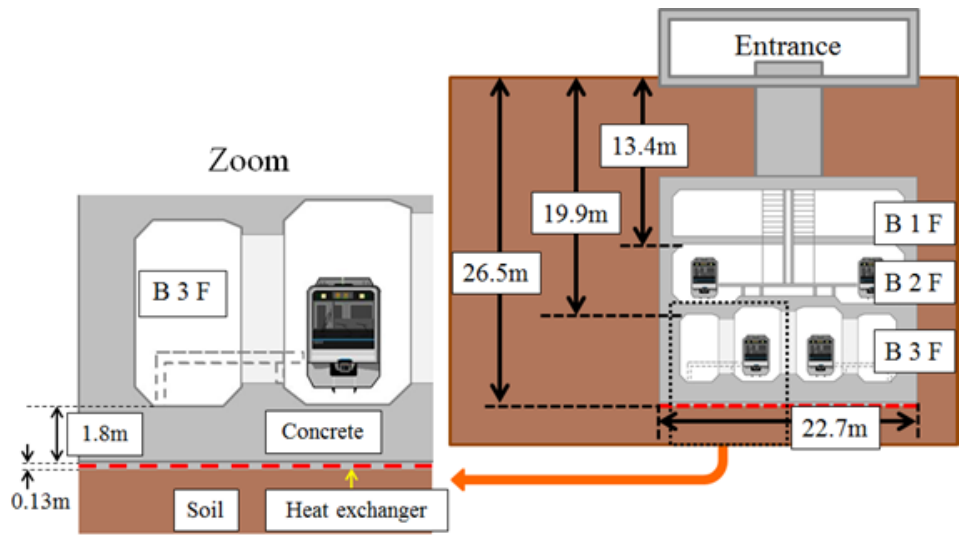

Figure 1 Cross sectional view of Setagaya-Daita Station.

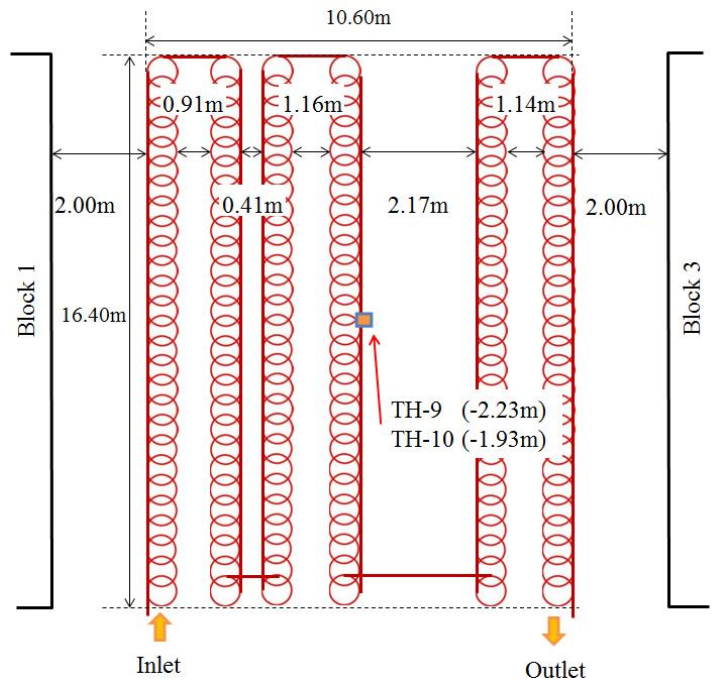

Figure 2 Layout of Slinky-coil GHEs

The HGHE fields are divided into 10 blocks each of which has a coil length of $100 \mathrm{~m}$ and pipe length of $500 \mathrm{~m}$ as shown in Figure 2; each heat pump is connected with two HGHE blocks. Water was used as the heat carrying fluid because there is no risk of freezing in the tunnel. In this research, Block No. 2 was chosen for the numerical modeling and the performance analysis since ground temperatures were measured only in this block. Two Pt100 temperature sensors were set in the central part of Block No.2, one at the depth pf GHE (TH-10) and one $0.3 \mathrm{~m}$ below the GHE (TH-9). The construction of the station was completed in the first half of 2013 and the operation of the GSHP system was started on July 3, 2013.

Figure 3 shows the temperature measured in $\mathrm{B} 2 \mathrm{~F}$, the local ambient temperature and the inlet and outlet temperatures of heat medium in Block No. 2 from July 2013 to July 2015. As shown in Figure3, the cooling operation started when the temperature in $\mathrm{B} 2 \mathrm{~F}$ exceeded $25^{\circ} \mathrm{C}$, while the heating operations were carried out when the temperature became below $17^{\circ} \mathrm{C}$. The yearly-average operation days of cooling and heating in the two years were 99 days and 151 days, respectively, which are significantly shorter than the normal cooling (135 days) and heating periods 
(161 days) in Tokyo. The shorter operation is explained by the stability of temperature in the tunnel in comparison with the ambient temperature. The daily operation hours of cooling and heating were 20 to 21 hours, which were much longer than the operation in buildings and houses, since the railway is operated from 5 A.M. to 1 A.M. The inlet temperature of heat medium to HGHE (outlet temperature of heat pumps) increased close to $40{ }^{\circ} \mathrm{C}$ in the cooling periods, which is not favorable to maintain high COP (Coefficient of Performance), while the temperature was maintained above $10{ }^{\circ} \mathrm{C}$ in each heating period.

Figure 4 shows the total heat exchange rate and COP of the heat pump in the same period. The average COP in the cooling and heating periods for two years were 4.65 and 3.05, respectively. In each period, the COP showed a steep decrease with the change of ground temperatures due to the long operation hours, low thermal conductivity of the ground and the lack of advection effects of groundwater flow in the concrete. The average heat exchange rates in the cooling and heating periods were calculated as $44.6 \mathrm{~kW}, 14.9 \mathrm{~kW}$, respectively, which could cause the rise of ground temperature in long-term operations. The comparison of COP in the first year and the second year, however, did not show a major reduction since cooling periods are much shorter than the heating periods and the difference between the cumulative heat exchange rates in the cooling $(6.34 \mathrm{MWh})$ and in the hearting periods $(4.15 \mathrm{MWh})$ were $53 \%$.

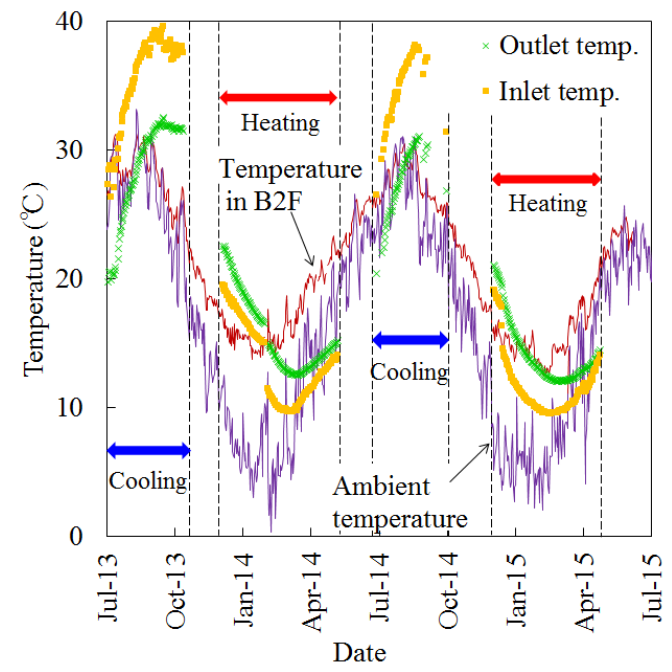

Figure 3 Measured temperature data in Block 2.

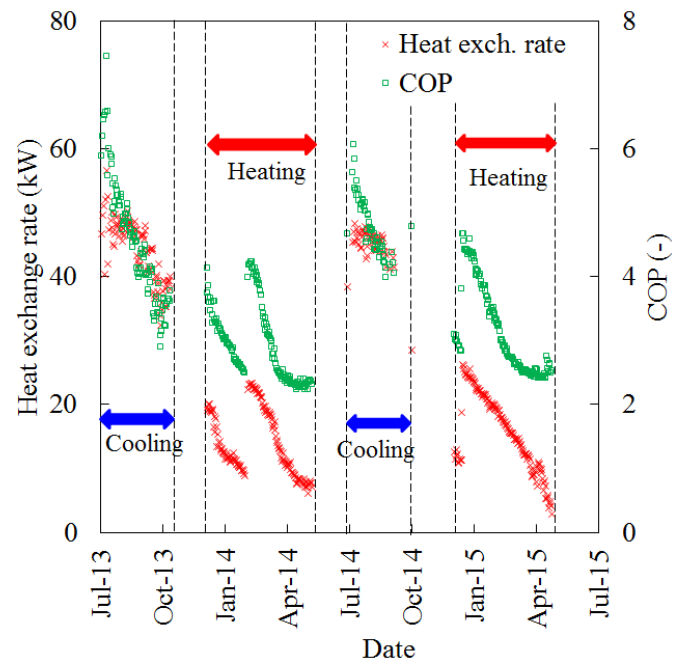

Figure 4 Measured COP performance.

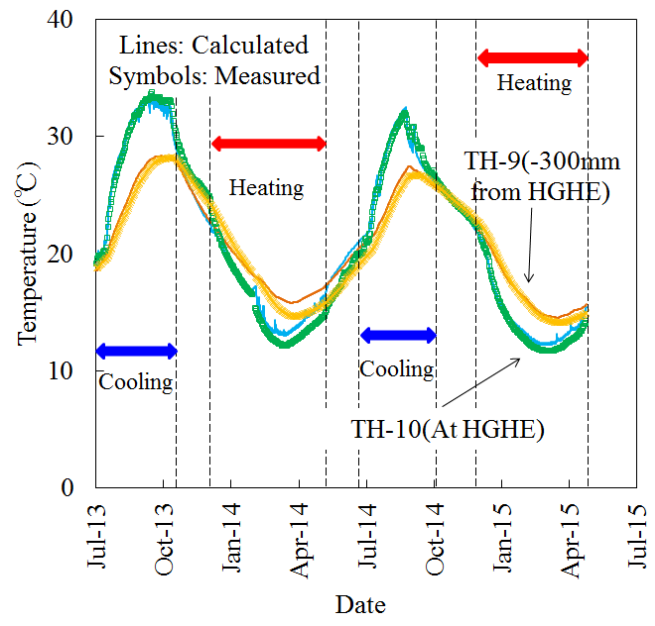

Figure 5 Measured and simulated ground temperatures in Block 2. 
In Figure 5, the changes of measured ground temperature at the depth pf GHE (TH-10) and $0.3 \mathrm{~m}$ below the GHE (TH-9) are shown with symbols. The temperature at the depth of the GHE showed a significant change due to the operation of the heat pumps; maximum $33.7^{\circ} \mathrm{C}$ in cooling and minimum $11.7^{\circ} \mathrm{C}$ in heating. At the beginning of the cooling period in June 2014, the ground temperature showed a recovery to the original temperature, while the temperature did not recover to the original temperature at the beginning of the heating period in December 2013 and December 2014. This indicates that the disposed heat in the cooling operations is stored in the ground due to the large cumulative heat disposal rate, while the cold heat has been dissipated in the ground after the heating seasons.

\section{NUMERICAL MODELING}

For the prediction of the performance of the HGHEs, a 3D numerical model was constructed for the HGHE and the surrounding ground using a finite-element based groundwater and thermal transport simulator, FEFLOW ver.7.0 (Diersch, 2014). Figure 6 shows the grid system of the numerical model. The area surrounded by red lines shows the position of the HGHEs. The heat carrying fluid flows from the inlet to the outlet of the HGHE. At the center of the HGHE, observation points are set to output the ground temperatures at the depth of the position of TH-9 and TH-10. The width of the upper and lower peripheral area of the model is defined short because adjacent blocks are located in this direction, which allows the use of adiabatic boundary conditions due to symmetry. To the right and left side of the model, $50 \mathrm{~m}$ of blank blocks are defined to avoid the interference of boundaries. The number of layers and the finite elements of each layer are set as 25 and 7,216, respectively. As shown in Figure 7, the spiral HGHEs are modeled using a thin flat plate based on the modeling approach of Fujii et al. (2012). The thermal conductivity of the polyethylene pipe was set as $0.03 \mathrm{~W} / \mathrm{K} / \mathrm{m}$ based on the loop pitch of $0.6 \mathrm{~m}$. The thickness of the flow path was defined as $0.0015 \mathrm{~m}$, which gives the same traveling time of heat carrying fluid from inlet to outlet. The initial temperature of the entire ground was set as $17.9^{\circ} \mathrm{C}$, which is $1.0^{\circ} \mathrm{C}$ higher than the annual mean temperature of Tokyo. The measured air temperature in the tunnel was used as the surface boundary condition, while the temperature at the bottom of the model was fixed at the initial temperature of $17.9^{\circ} \mathrm{C}$. The thickness of the model was defined as $10 \mathrm{~m}$ to eliminate the effect of the bottom boundary condition.

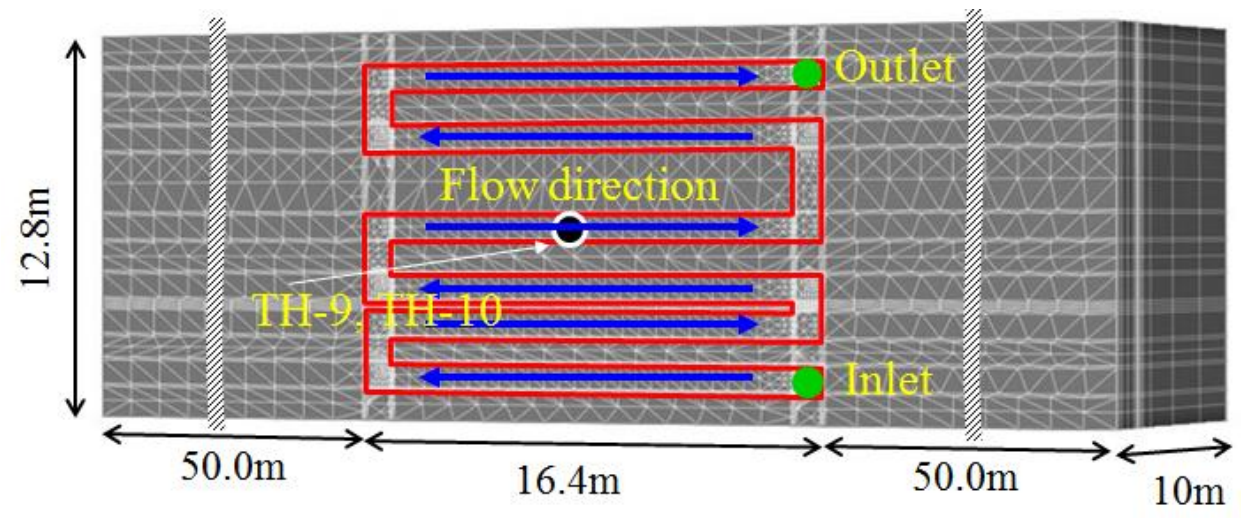

Figure $63 \mathrm{D}$ view of the numerical simulation model. 


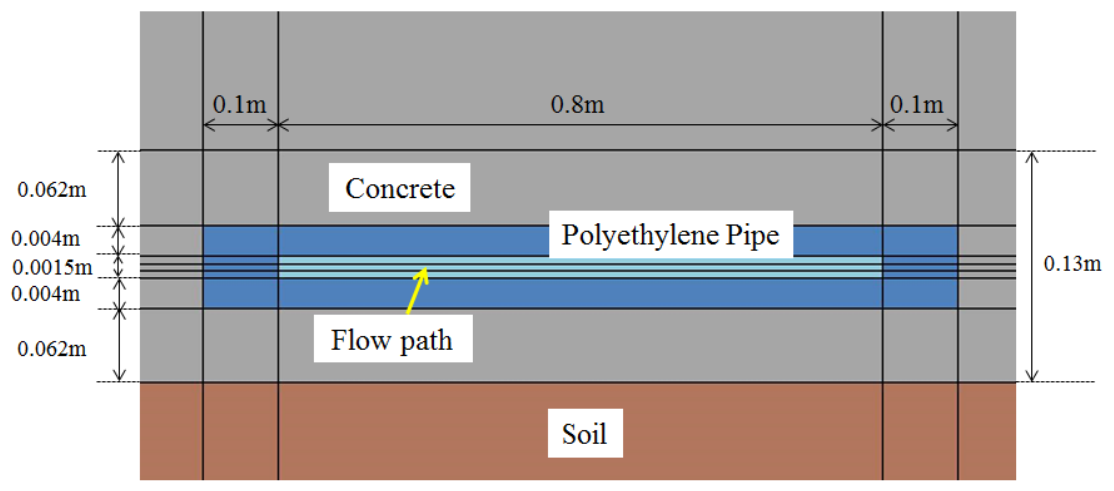

Figure 7 Treatment of Slinky-coils in the numerical model.

The model was run for the two years' cooling and heating operations, treating the inlet temperature as the input data. The model was validated through the history matching between the measured and simulated temperatures. The history matching results of ground temperatures is shown in Figure 5. Reasonably good agreement was obtained on the measured and simulated ground temperature except in the period between January and April, 2014. The reason of the disagreement was considered as the malfunction of data acquisition system during this period. The problem was fixed in April, 2014 and reasonable history matching results were obtained afterwards. The Root Square Mean Error (RSME) between the measured and simulated temperatures at TH-10 and TH- 9 are $0.94{ }^{\circ} \mathrm{C}$ and $0.85^{\circ} \mathrm{C}$, respectively during the 2 years of operation. The RMSE is reduced to $0.82{ }^{\circ} \mathrm{C}$ and $0.71{ }^{\circ} \mathrm{C}$, respectively, excluding the period between January and April, 2014. Figure 8 shows the measured and simulated outlet temperatures of heat medium. Though the matching results were poor between January and April, 2014 as mentioned above, good matching results were obtained for the rest of the cooling and heating periods. The RSME between the measured and simulated outlet temperatures are $0.71{ }^{\circ} \mathrm{C}$ during the 2 years of operation excluding the period between January and April, 2014.

In the above history matching simulations, the validity of the numerical model was sufficiently demonstrated. The following section discusses the optimum design of the HGHEs using the validated numerical model.

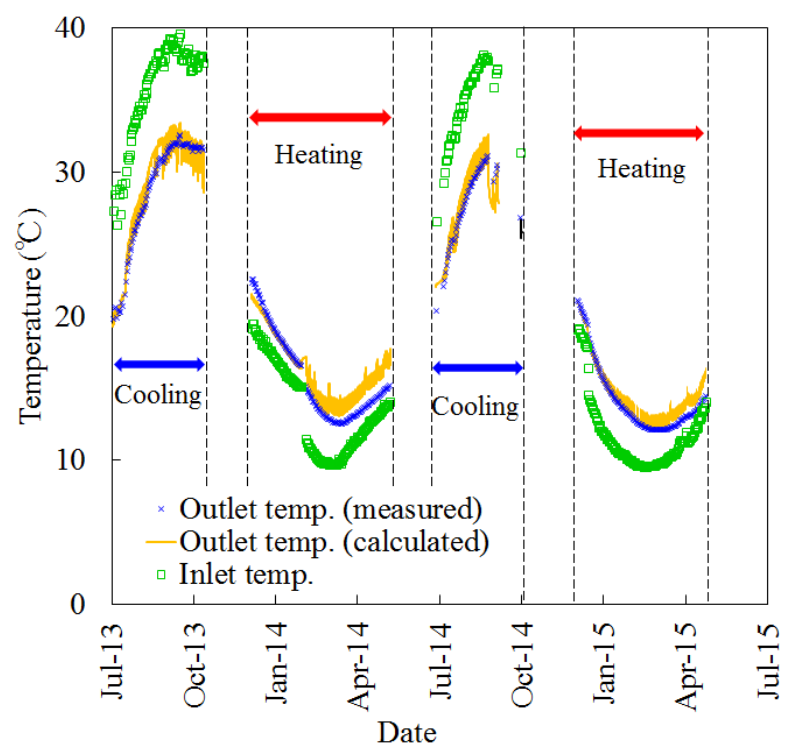

Figure 8 Measured and simulated outlet temperatures in Block 2. 


\section{SENSITIVITY STUDIES}

In this section, sensitivity studies are performed using the numerical model to optimize the design and operation strategy of the GSHP system based on the long-term prediction. The heating period was fixed as 5 months, from November to April in each year, while the length of the cooling period was set as 2 months (July - August, Case 1) or 4 months (June - September, Case 2) in the prediction terms of 10 years. The size of the HGHE field was set equal to the Block No. 2 in the train station, though the influence of the adjacent block were not considered in the sensitivity study. The cooling and heating loads for the field were set as $4.46 \mathrm{~kW}$ and $1.49 \mathrm{~kW}$, respectively, based on the actual operations. The total heat extraction rate of 1 year is calculated as $4.50 \mathrm{MWh}$, while the rejection rate in the same period are 5.53MWh and 10.88MWh in Case1 and Case 2, respectively. The poor balance of heat exchange rate, especially in Case 2, could cause the increase of ground temperature and the reduction of COP in long-term operations.

Figure 9 shows the predicted outlet temperature of heat carrying fluid from the HGHE obtained in the simulation. In the first 2 years, the temperatures show a small drop in both cases, but they get stabilized for the rest of the simulation even in Case 2. This suggests that the HGHEs in the tunnel can maintain stable heat exchange performance even when the heat balance is not well maintained. The heat flow from the bottom of the HGHEs and the heat exchange at the surface of the tunnels will be the main energy source of temperature recovery during the periods between the cooling and heating seasons.

Figure 10 shows the contour map of the temperature in the ground at the depth of the HGHE in Case 2. The three figures show the temperature at the beginning of the heating periods in the (a) 1st, (b) 2nd and (c) 10th operation years, respectively. The central part of the figure shows the HGHE field. The comparison of the distribution in the first year and the second years indicates the temperature drop of about $1{ }^{\circ} \mathrm{C}$ in the HGHE field, while the distribution in 10th years is quite similar to the one in the second year. These figures demonstrate the stable performance of the GSHP system using the tunnels even in the case of poor energy balance.

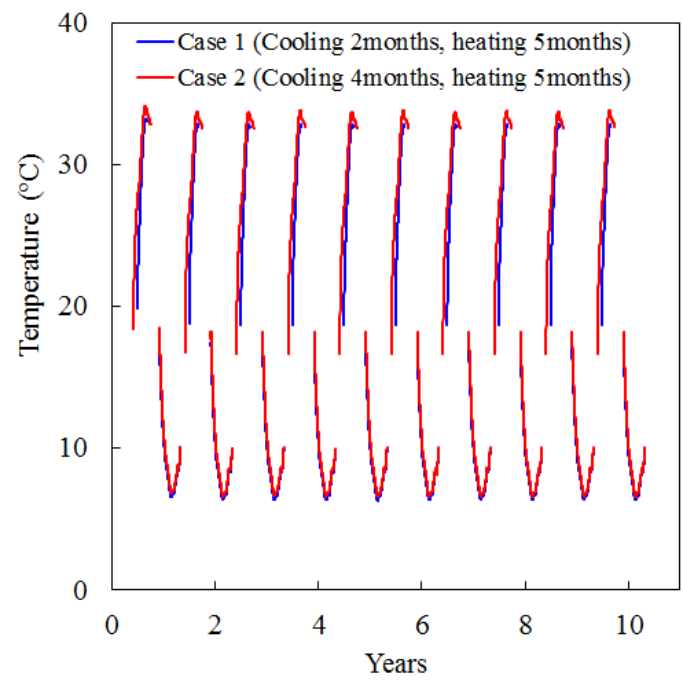

Figure 9 Prediction results of outlet temperature of heat carrying fluid in Case 1 and Case 2 
Case 2 (Cooling 4months, heating 5months)
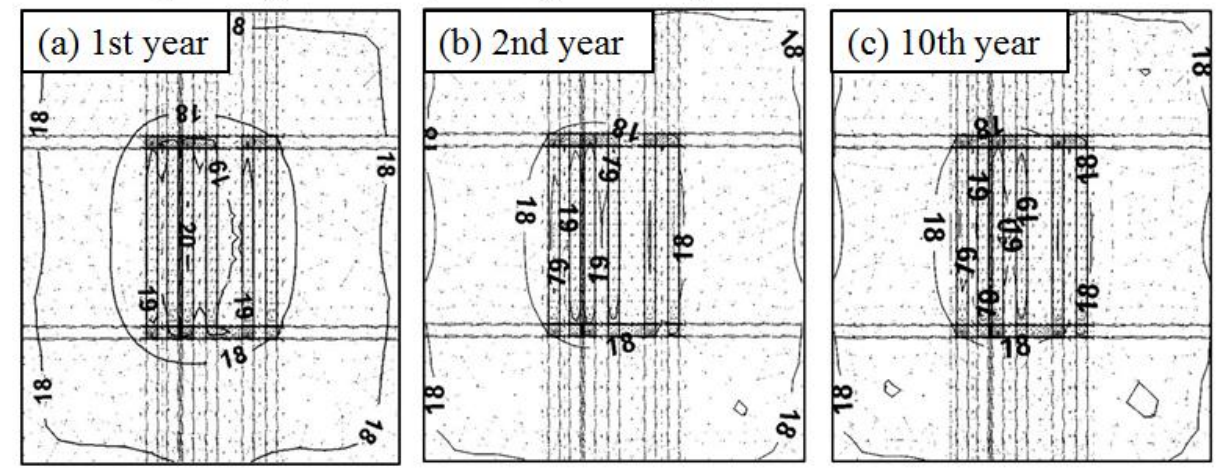

Figure 10 Contour maps of simulated temperatures at the depth of HGHEs at the beginning of the heating periods of (a) 1st, (b) 2nd and (c) 10th years.

Next, the effect of the burial depth of the HGHE was examined by changing the depth as $1 \mathrm{~m}, 2 \mathrm{~m}$ and $3 \mathrm{~m}$ below the surface of the tunnel. The heating period was fixed as 5 months from November to April and the cooling period was set as 4 months from June to September. Same heat exxhange rates were applied as used in Case 2. Figure 11 shows the season-averaged outlet temperature of the heat medium from the HGHE in each case. In all cases, the temperatures get stabilized within 3 years indicating the stabilization of the ground temperatures. In the cooling and heating operations, the difference in the heat medium temperature between the $1 \mathrm{~m}$ and $3 \mathrm{~m}$ were $1.4{ }^{\circ} \mathrm{C}$ and $3.4^{\circ} \mathrm{C}$. This suggests that the deeper installation of HGHE is more important in the heating operation than in the cooling operation. The difference can be explained by the difference in heat exchange rates in the cooling operation (25.7 $\left.\mathrm{W} / \mathrm{m}^{2}\right)$ and heating operations $\left(8.6 \mathrm{~W} / \mathrm{m}^{2}\right)$. In the cooling operation, larger heat exchange rate between the air and the ground is expected at the surface of the tunnel due to the larger heat exchange rate per unit area, while most of the heat is supplied from the ground when the HGHE is deep. In the case of heating operations, the small heat exchange rates increase the ratio of heat from the bottom, which clearly shows the effect of installation depth.

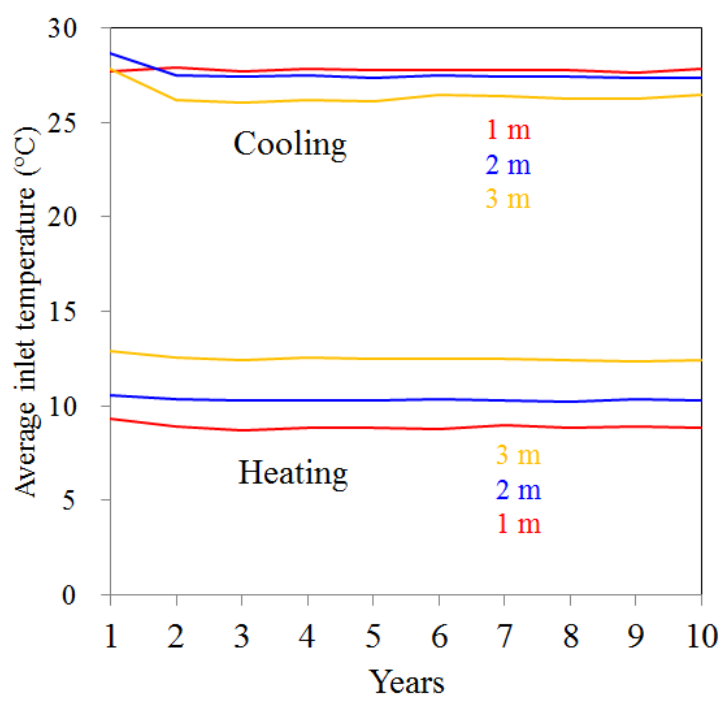

Figure 11 Simulated season-averaged temperature of heat medium at the outlet of HGHEs. 


\section{CONCLUSION}

The interpretation of operation data and numerical modeling were carried out in a GSHP system using Slinkycoil HGHEs installed in the basement of a railway tunnel in Tokyo, Japan. The interpretation of the operation data for 2 years demonstrated the stable energy efficiency of the system, though the amount of heat release and extraction were not well balanced in the GSHP system. A numerical simulation model of the HGHE was then developed based on the physical properties of the ground and the design of the HGHEs. The model was well-validated using the monitored data during the heating and cooling operations. The sensitivity studies using the model showed that the GSHP system will maintain the initial heating and cooling capacity for 10 years and the deeper installation of the HGHE is preferable to obtain higher COP, especially in the heating operation.

\section{ACKNOWLEDGMENTS}

The authors would like to thank Odakyu Electric Railway Co., Ltd. for providing the operation data of the GSHP system. This work was partly supported by Grants-in-Aid for Scientific Research (B) (JP15H04223) from JSPS.

\section{REFERENCES}

Barla, M., Di Donna, A., Perino, A. 2016. Application of energy tunnels to an urban environment. Geothermics 61: 104-113.

Bourne-IWebb, P., Freitas, T., Gonçalves, R. 2016. Thermal and mechanical aspects of the response of embedded retaining walls used as shallow geothermal heat exchangers. Energy and Buildings 125: 130-141.

Diersch, H.J.G. 2014. FEFLOW Finite Element Modeling of Flow, Mass and Heat Transport in Porous and Fractured Media. Springer, $996 \mathrm{p}$.

Frodl, S., Franzius, J., Bartl, T. (2010) Design and construction of the tunnel geothermal system in Jenbach. Geomechanics and Tunnelling, 3, 658-668.

Fujii, H., Nishi, K., Komaniwa, Y., Chou, N. 2012. Numerical modeling of slinky-coil horizontal ground beat exchangers. Geothermics 41: 55-62.

Nicholson, D.P., Chen, Q., Pillai, A., Chendorain, M. 2013. Development in the thermal pile and thermal tunnel linings for city scale GSHP systems. 38th Stanford Geothermal Workshop SGP-TR-198. 